Research Journal of Applied Sciences, Engineering and Technology 5(2): 654-658, 2013

DOI:10.19026/rjaset.5.5004

ISSN: 2040-7459; E-ISSN: 2040-7467

(C) 2013 Maxwell Scientific Publication Corp.

Submitted: June 02, $2012 \quad$ Accepted: July 09, $2012 \quad$ Published: January 11, 2013

Research Article

\title{
Multi-level Extensible Synthetic Evaluation of the Quality of Passenger Transport Service of High-speed Rail
}

\author{
${ }^{1}$ Yanbing Fu, ${ }^{1}$ Zhiya Chen and ${ }^{2}$ Xiujie Zhao \\ ${ }^{1}$ School of Traffic and Transportation Engineering, Central South University, Changsha 410075, China \\ ${ }^{2}$ Nanning Survey and Design Institute Coporation Limited of China Railway Siyuan \\ Group, Nanning 530003, China
}

\begin{abstract}
This study establishes the evaluation index system of the quality of passenger transport service of highspeed rail based on the characteristics of passenger transport and determines the index weights with the Fuzzy Analytic Hierarchy Process. In order to make up for the deficiencies of present quality evaluation method of passenger transport service of high-speed rail, this study develops the multi-level extensible synthetic evaluation model of the quality of passenger transport service of high-speed rail with the core of extension method based on the questionnaire survey to the passengers. This study tests the model with the data surveyed in a passenger station of high-speed rail, which can not only expand extension application areas, but also provide new ideas and means for the evaluation of the quality of passenger transport service of high-speed rail.
\end{abstract}

Keywords: Extension, high-speed rail, passenger transport, quality of service, synthetic evaluation

\section{INTRODUCTION}

As a kind of safe and reliable, low-carbon and environment-protection, large carrying-capacity, fast and comfortable transport mode, high-speed rail has become an important trend of rail. At present, China is the country with the longest operation mileage of highspeed rail in the world and the passengers are putting forwards higher demands for the quality of service. So it is very useful to evaluate the quality of the passenger transport service of high-speed rail and to enhance the level of service.

Recently, some scholars do the research on the scope, contents, index system and the method of the evaluation of the quality of passenger transport service and have made some progress. Literature (Hitoshi et al., 2010) analyzed the prerequisite conditions and policy of the impact of the quality of rail services. Literature (Cao and Chen, 2012) analyzed the relationship of service quality, passenger complaints and passenger loyalty based on the Structural Equation Model (SEM). Literature (Xuan, 2010) established the evaluation index system of the quality of passenger transport service and constructed the evaluation model considering the differences of the design quality and technology quality. Literature (Liu, 2007) defined the synthetic evaluation indexes of the passenger transport service.

In this study, we establish the evaluation index system of the quality of passenger transport service of high-speed rail based on the characteristics of passenger transport and determine the index weights with the Fuzzy Analytic Hierarchy Process. In order to make up for the deficiencies of present quality evaluation method of passenger transport service of high-speed rail, this study develops the multi-level extensible synthetic evaluation model of the quality of passenger transport service of high-speed rail with the core of extension method based on the questionnaire survey to the passengers. This study tests the model with the data surveyed in a passenger station of high-speed rail, which can not only expand extension application areas, but also provide new ideas and means for the evaluation of the quality of passenger transport service of high-speed rail.

\section{EVALUATION INDEX SYSTEM}

Characters of the quality of passenger transport service of high-speed rail: Based on the definition of quality of passenger transport service, there are serial main characters which are as follows:

Safety: To ensure the train operation safety is the essential task for the enterprise of passenger transport of high-speed rail and passengers often select such transport mode which is more safety in their mind, according to their experience. And it includes

\footnotetext{
Corresponding Author: Yanbing Fu, School of Traffic and Transportation Engineering, Central South University, Changsha 410075, China

This work is licensed under a Creative Commons Attribution 4.0 International License (URL: http://creativecommons.org/licenses/by/4.0/).
} 


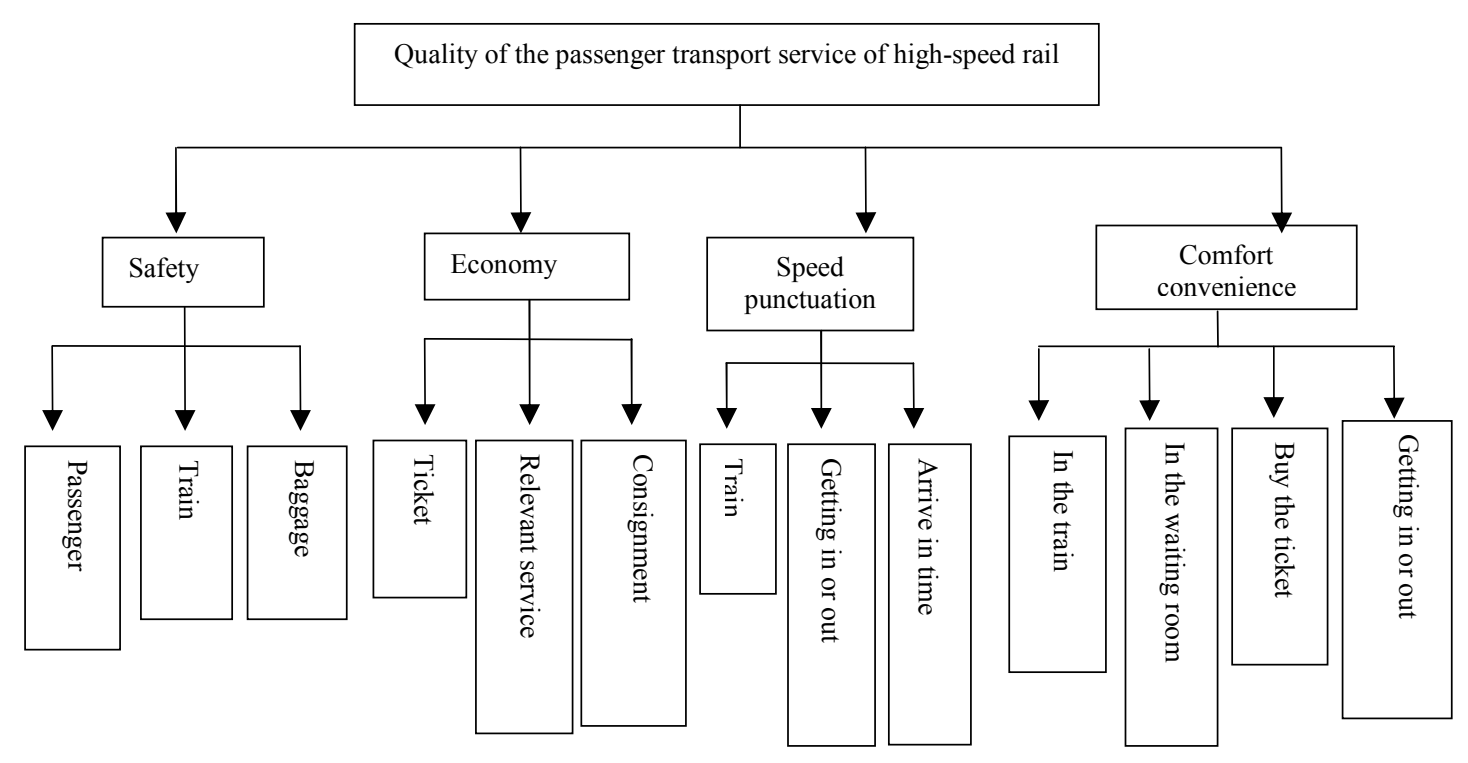

Fig. 1: Evaluation index system

the safety of passengers, the safety of trains and the safety of baggage.

Economy: Economy refers to that the ticket price and service charge is fair and reasonable, which is the main influence factor when the passengers are in the choice of the transport mode. It includes the price of ticket, the price of relevant service and the price of baggage consignment.

Speed and punctuation: Speed is the hallmark of the progress of traffic science and technology and to arrive at their destination on time is one of the basic requirements of the passengers. The travel time is the important factor when the passengers evaluate the quality of their journey. It includes the speed of trains, the speed of passengers when their getting in or out of the station and the train-arriving in time.

Comfort and convenience: Comfort mainly refers to that the integrated feeling about the service of the transport enterprise of the passengers in the course of their journey. With the improvement of the livingstandard of people and the development of transport industry, passengers have put forward higher and higher requirements on the comfort of their journey. And convenience refers to that it will be very convenient and fast to buy the tickets, to take the train and to transfer. It includes comfort in the train and in the waiting room and convenience when buying the ticket, when getting in or out of the station.

Evaluation index system: According to the aboveanalysis, we can construct the evaluation index system of quality of passenger transport service of highspeed rail, which is shown in Fig. 1.

\section{MULTI-LEVEL EXTENSIBLE COMPREHENSIVE EVALUATION MODEL}

The evaluation of quality of passenger transport service of high-speed rail includes multiple evaluation indexes, among which there are some subordination relations. So this study will evaluate the quality of passenger transport service of high-speed rail with the theory of matter elements and extension method and the steps are as follows:

To determine the weight of evaluation index: In order to make it understood all the variables with the superscripts of 0 are the primary evaluation indexes, which are denoted as the indexes of 0 . All the variables with the superscripts of 1 are the senior evaluation indexes which are relevant to the index of safety and which are denoted as the indexes of 1 and then 2 represents this kind of indexes are relevant to the index of economy and 3 represents this kind of indexes are relevant to the index of speed and punctuation and 4 represents this kind of indexes are relevant to the index of comfort and convenient.

This study determines the index weights with fuzzy analytic hierarchy method and the steps are as follows:

First, to construct the evaluation index set accord to the Table 1 which is denoted as:

$$
U^{d}=\left[c_{1}^{d}, c_{2}^{d}, \cdots, c_{d_{n}}{ }^{d}\right], d=1,2,3,4
$$


Table 1: The index system and their weight

\begin{tabular}{lllll}
\hline Primary index & Weight & Senior index & Weight & Score \\
\hline$C_{1}{ }^{0}$ & 0.3931 & $C_{1}{ }^{1}$ & 0.5132 & 96.2 \\
& & $C_{2}{ }^{1}$ & 0.4029 & 95.9 \\
$C_{2}{ }^{0}$ & & $C_{3}{ }^{1}$ & 0.0839 & 93.9 \\
& \multirow{2}{*}{0.2802} & $C_{1}{ }^{2}$ & 0.6115 & 56.5 \\
$C_{3}{ }^{0}$ & & $C_{2}{ }^{2}$ & 0.2926 & 60.3 \\
& \multirow{3}{*}{0.1651} & $C_{3}{ }^{2}$ & 0.0961 & 53.3 \\
$C_{4}{ }^{0}$ & & $C_{1}{ }^{3}$ & 0.6812 & 95.6 \\
& & $C_{2}{ }^{3}$ & 0.1925 & 93.2 \\
& \multirow{2}{*}{0.1216} & $C_{3}{ }^{3}$ & 0.1263 & 93.9 \\
& & $C_{1}{ }^{4}$ & 0.5169 & 88.1 \\
& & $C_{2}{ }^{4}$ & 0.1530 & 85.2 \\
& & $C_{3}{ }^{4}$ & 0.2186 & 86.2 \\
& & $C_{4}{ }^{4}$ & 0.1125 & 83.3 \\
\hline
\end{tabular}

Second, to retain some experts (the quantities of them are denoted as s) to score the six indexes and we can get $\mathrm{s} d_{n} \times d_{n}$ fuzzy evaluation matrixes, which are denoted as:

$$
A_{m}{ }^{d}=\left[\begin{array}{ccc}
a_{m 11}{ }^{d} & \mathrm{~L} & a_{m 1 d_{n}{ }^{d}} \\
\mathrm{M} & & \mathrm{M}{ }_{d} \\
a_{m d_{n} 1}{ }^{d} & \mathrm{~L} & a_{m d_{n} d_{n}}{ }^{d}
\end{array}\right], \quad m=1,2, \mathrm{~L}, s
$$

and $m$ represents the no. $m$ expert.

Third, to determine the weights of those experts, which are denoted as $\lambda_{1}, \lambda_{2},\left\llcorner\lambda_{\mathrm{s}}\right.$ Fourth, to summary those experts' opinions and then we can get the synthesis judgment matrix $\bar{A}^{2}=\left(\bar{a}_{i j}\right)_{d_{n} \times d_{n}}{ }^{d}$, after the matrix transformation, we will obtain fuzzy consistent matrix $r^{d}=\left(r_{i j}\right)_{d_{n} \times d_{n}}{ }^{d}$ and then we can get the index weights vector which is denoted as:

$$
W^{d}=\left(w_{1}^{d}, w_{2}^{d}, \mathrm{~L}, w_{d_{n}}{ }^{d}\right)
$$

To build the evaluation grade: Based on the requirements of the evaluation, this study sets up the evaluation grade of those evaluation indexes, which is denoted as $\mathrm{N}=\left(\mathrm{N}_{\mathrm{k}}\right)=\left(\mathrm{N}_{1}, \mathrm{~N}_{2}, \mathrm{~L}, \mathrm{~N}_{1}\right), 1$, represents the quantities of the evaluation grade and $N_{k}, k=1,2, L, 1$ represents the evaluation grade of $k$. This study uses the 5-level remark collective for all kinds of evaluation index (Feng, 2011), that is $\mathrm{V}=$ (very satisfied, satisfied, basic satisfied, unsatisfied, very unsatisfied) and then determines the data range of all the evaluation grades, according to the actual situation and the point of views of the experts and scholars.

To design the questionnaire: The questionnaire includes the above six evaluation indexes and determines that the score range of each index is between 50 to 100 and the index of very satisfied is between 90 to 100 and the index of satisfied is between 80 to 90 and the index of basic satisfied is between 70 to 80 and the index of unsatisfied is between 60 to 70 and the index very unsatisfied is between 50 to 60 .

To determine the classical domain of the evaluation grade: Classical domain refers to the data range of 1 of the evaluation index, which is denoted as:

$$
\begin{aligned}
& R_{k}{ }^{d}=\left(N_{k}{ }^{d}, c_{i}{ }^{d}, v_{i k}{ }^{d}\right)=\left[\begin{array}{cccc}
N_{k}{ }^{d} & c_{1}{ }^{d} & v_{1 k}{ }^{d} \\
& c_{2}{ }^{d} & v_{2 k}{ }^{d} \\
& \vdots & \vdots \\
& \\
& c_{n}{ }^{d} & v_{n k}{ }^{d}
\end{array}\right] \\
& =\left[\begin{array}{ccc}
N_{k}{ }^{d} c_{1}{ }^{d} & <a_{1 k}{ }^{d}, b_{1 k}{ }^{d}> \\
& c_{2}{ }^{d} & <a_{2 k}{ }^{d}, b_{2 k}{ }^{d}> \\
\vdots & \vdots \\
c_{n}{ }^{d} & <a_{n k}{ }^{d}, b_{n k}{ }^{d}>
\end{array}\right]
\end{aligned}
$$

and $\mathrm{R}_{\mathrm{k}}^{\mathrm{d}}$, $=1,2$, $L, 1$ represents the classical domain of those evaluation indexes related to the evaluation grade of $\mathrm{k}$ and $\mathrm{c}_{\mathrm{k}}^{\mathrm{d}}, \mathrm{i}=1,2, \mathrm{~L}, \mathrm{n}$ represents the evaluation index of $\mathrm{i}$ and $\mathrm{v}_{\mathrm{k}}^{\mathrm{d}}, \mathrm{i}=1,2, L, \mathrm{n}$ represents the range of $\mathrm{N}^{\mathrm{d}}{ }_{\mathrm{k}}$ related to $\mathrm{c}^{\mathrm{d}}{ }_{\mathrm{i}}$ and $\left\langle a_{i k}^{d}, b_{i k}^{d}>, i=1,2, \mathrm{~L}, n\right.$ is its data of the range and $\mathrm{d}, \mathrm{I}=1,2,\llcorner, 6$.

To determine the joint domain of the evaluation index: Joint domain refers to all the data range of one of the evaluation index, which is denoted as:

$$
\begin{aligned}
& R_{q}{ }^{d}=\left(N_{q}{ }^{d}, c_{i}{ }^{d}, v_{i q}{ }^{d}\right)=\left[\begin{array}{ccc}
N_{q}{ }^{d} & c_{1}{ }^{d} & v_{1 q}{ }^{d} \\
& c_{2}{ }^{d} & v_{2 q}{ }^{d} \\
\vdots & \vdots \\
& \\
& c_{n}{ }^{d} & v_{n q}{ }^{d}
\end{array}\right] \\
& =\left[\begin{array}{cc}
N_{k}{ }^{d} c_{1}{ }^{d} & <a_{1 q}{ }^{d}, b_{1 q}{ }^{d}> \\
c_{2}{ }^{d} & <a_{2 q}{ }^{d}, b_{2 q}{ }^{d}> \\
\vdots & \vdots \\
c_{n}{ }^{d} & <a_{n q}{ }^{d}, b_{n q}{ }^{d}>
\end{array}\right]
\end{aligned}
$$

and $\mathrm{R}_{\mathrm{q}}^{\mathrm{d}}$ represents the joint domain and $\mathrm{R}_{\mathrm{q}}^{\mathrm{d}}$ represents all the evaluation grades and $v_{i q}^{d}, i=1,2,\llcorner, n$ represents the range of $\mathrm{N}_{\mathrm{q}}^{\mathrm{d}}$ and $\left\langle a_{i q}^{d}, b_{i q}^{d}\right\rangle, i=$ $1,2,\llcorner, n$ is its data of the range and $d, i=1,2,\llcorner, 6$.

To determine evaluation matter element to will be evaluated: To put the related data into the matter element model, this is denoted as: 


$$
R^{d}=\left(P^{d}, c_{i}{ }^{d}, x_{i}{ }^{d}\right)=\left[\begin{array}{ccc}
P^{d} & c_{1}{ }^{d} & x_{1}{ }^{d} \\
& c_{2}{ }^{d} & x_{2}{ }^{d} \\
& \mathrm{M} & \mathrm{M} \\
& c_{n}{ }^{d} & x_{n}{ }^{d}
\end{array}\right]
$$

among which $R^{d}$ represents the matter element will be evaluated and $\mathrm{P}^{\mathrm{d}}$ represents the object will be evaluated, namely the quality of passenger transport service of the high-speed rail and $\mathrm{x}_{\mathrm{i}}^{\mathrm{d}}, \mathrm{i}=1,2, L, \mathrm{n}$ is the data of $\mathrm{P}^{\mathrm{d}}$ related to the evaluation index of $i$ and $d, i=1,2,\llcorner, 6$.

To calculate the function values of interrelatedness of the second indexes: To put that:

$$
e_{k}{ }^{d}\left(x_{i}{ }^{d}\right)=\left\{\begin{array}{l}
-\frac{\rho^{d}\left(x_{i}{ }^{d}, v_{i k}{ }^{d}\right)}{\left|v_{i k}\right|}, \\
x_{i}{ }^{d} \in v_{i k}{ }^{d} \\
-\frac{\rho^{d}\left(x_{i}{ }^{d}, v_{i k}{ }^{d}\right)}{\rho^{d}\left(x_{i}{ }^{d}, v_{i q}{ }^{d}\right)-\rho^{d}\left(x_{i}{ }^{d}, v_{i k}{ }^{d}\right)}, \\
x_{i}{ }^{d} \notin v_{i k}{ }^{d}
\end{array}\right.
$$

Among which,

$$
\begin{aligned}
& \rho^{d}\left(x_{i}{ }^{d}, v_{i k}{ }^{d}\right)=\left|x_{i}{ }^{d}-\frac{1}{2}\left(a_{i k}{ }^{d}+b_{i k}{ }^{d}\right)\right| \\
& -\frac{1}{2}\left(b_{i k}{ }^{d}-a_{i k}{ }^{d}\right) \\
& \rho^{d}\left(x_{i}{ }^{d}, v_{i q}{ }^{d}\right)=\mid x_{i}{ }^{d}-\frac{1}{2}\left(a_{i q}{ }^{d}+b_{i q}{ }^{d}\right) \\
& \mid-\frac{1}{2}\left(b_{i q}{ }^{d}-a_{i q}{ }^{d}\right)
\end{aligned}
$$

And, $d, i=1,2, \mathrm{~L}, 6$

\section{The primary extensible synthetic evaluation:} To put that:

$$
\begin{aligned}
& g_{k}{ }^{d}\left(P^{d}\right)=\sum_{i=1}^{n} w_{i}{ }^{d} \cdot e_{k}{ }^{d}\left(x_{i}{ }^{d}\right) \\
& G^{0}=\left[\begin{array}{ccc}
g_{1}{ }^{1}\left(P^{1}\right) & \mathrm{L}_{5}{ }^{1}\left(P^{1}\right) \\
\mathrm{M} & \mathrm{M} \\
g_{1}{ }^{6}\left(P^{6}\right) & \mathrm{L} & g_{5}{ }^{6}\left(P^{6}\right)
\end{array}\right]
\end{aligned}
$$

among which, $e_{k}^{d}\left(x_{\dot{j}}^{d}\right)$ represents the function value of interrelatedness of $\mathrm{P}^{\mathrm{d}}$ related to the evaluation grade of $\mathrm{k}$ and $\mathrm{g}_{k}^{\mathrm{d}}\left(\mathrm{P}^{\mathrm{d}}\right)$ represents the interrelatedness of $\mathrm{P}^{\mathrm{d}}$ related to the evaluation grade of $k$ and $\mathrm{G}^{0}$ is a matrix of $6 \times 5$, which is constructed by the interrelatedness between all kinds of second grade indexes and the 5 evaluation grades.

\section{The senior extensible synthetic evaluation:}

To put that:

$$
g_{k}{ }^{0}\left(P^{0}\right)=\sum_{i=1}^{n} w_{i}^{0} \cdot G^{0}
$$

among which, $\mathrm{g}_{\mathrm{k}}{ }^{\mathrm{O}}\left(\mathrm{p}^{\mathrm{o}}\right)$ represent the interrelated nesses of $\mathrm{p}^{\mathrm{o}}$ related to the evaluation grade of $\mathrm{k}$ and are the final evaluation values.

To evaluate the grade of the quality of passenger transport service of high-speed rail: To compare the calculation results with the interrelatedness of all the evaluation grade set and the smaller the interrelatedness is and the smaller the adaptability between $\mathrm{P}^{0}$ and one evaluation grade set and vice versa. To put that:

$$
h_{k}=\max g_{k}^{0}\left(P^{0}\right)
$$

and $h_{k}$ represents the maximum value of the interrelatedness between $\mathrm{p}^{\circ}$ and 1 evaluation grades.

If $h_{k}>0$, then $\mathrm{P}^{0}$ belongs to the evaluation grade of $k$ and if $h_{k}>0$, then $\mathrm{P}^{0}$ doesn't belong to all the evaluation grade, which should be cast out.

\section{EXAMPLE ANALYSIS}

This study takes the second indexes of ticket price, relevant service price and baggage consignment price which belong to the index of economy in the first grade as an example to describe the calculation and application of the synthetic extension evaluation model of quality of passenger transport service of high-speed rail based on matter element theory and extension theory.

The weight of the evaluation index: Ten experts are retained to determine the score of the above evaluation indexes which describe the quality of passenger transport service of high-speed rail and other experts are retained to determine the weight of those experts. According to the steps described in 3.1, we can calculate the vector of all the index weights which is listed in Table 1.

Questionnaires and data statistics: We collected the evaluation information of the passenger on the quality of passenger transport service of high-speed rail by means of questionnaire at the station exit, entrance and waiting room of a high-speed rail. 550 questionnaires are 
distributed among which 531 questionnaires are usable. The statistics data of the matter element of the investigation is shown in Table 1:

Classical domain: According to the questionnaires, the classical domains of the five evaluation grades of all the evaluation indexes are respectively $<90,100\rangle,<80,90>$, $<70,80>,<60,70>$ and $<50,60>$. For instance:

$$
\begin{aligned}
& R_{1}^{2}=\left[\begin{array}{lll}
\text { very } & c_{1}{ }^{2} & <90,100> \\
\text { satisfied } & c_{2}{ }^{2} & <90,100> \\
& c_{3}{ }^{2} & <90,100>
\end{array}\right] \\
& R_{2}{ }^{2}=\left[\begin{array}{ccc}
\text { satisfied } & c_{1}{ }^{2} & <90,100> \\
& c_{2}{ }^{2} & <90,100> \\
& c_{3}{ }^{2} & <90,100>
\end{array}\right] \\
& R_{3}^{2}=\left[\begin{array}{lll}
\text { basic } & c_{1}{ }^{2} & <70,80> \\
\text { satisfied } & c_{2}{ }^{2} & <70,80> \\
& c_{3}{ }^{2} & <70,80>
\end{array}\right] \\
& R_{4}{ }^{2}=\left[\begin{array}{ccc}
\text { unsatisfied } & c_{1}{ }^{2} & <60,70> \\
& c_{2}{ }^{2} & <60,70> \\
& c_{3}{ }^{2} & <60,70>
\end{array}\right] \\
& R_{5}^{2}=\left[\begin{array}{lll}
\text { very } & c_{1}^{2} & <50,60> \\
\text { unsatisfied } & c_{2}^{2} & <50,60> \\
& c_{3}^{2} & <50,60>
\end{array}\right]
\end{aligned}
$$

Joint domain: According to the questionnaires, the joint domain of the evaluation grades of all the evaluation indexes is $<50,100\rangle$. For instance:

$$
R_{q}^{2}=\left[\begin{array}{ccc}
N_{q}^{2} & c_{1}^{2} & <50,100> \\
& c_{2}^{2} & <50,100> \\
& c_{3}^{2} & <50,100>
\end{array}\right]
$$

Evaluation matter element will be evaluated: According to the questionnaires, we can get the evaluation matter element will be evaluated. For instance:

$$
R^{2}=\left[\begin{array}{ccc}
P^{2} & c_{1}^{2} & 59.5 \\
& c_{2}^{2} & 62.3 \\
& c_{3}^{2} & 55.3
\end{array}\right]
$$

The senior extensible evaluation: According to the steps described above, we can calculate that:

$$
\begin{aligned}
& g_{1}{ }^{0}\left(P^{0}\right)=0.03, g_{2}{ }^{0}\left(P^{0}\right)=0.22, g_{3}{ }^{0}\left(P^{0}\right)=-0.25, \\
& g_{4}{ }^{0}\left(P^{0}\right)=-0.32, g_{5}{ }^{0}\left(P^{0}\right)=-0.59
\end{aligned}
$$

Grade evaluation: According to the evaluation criteria, $\mathrm{h}_{2}=0.22>0$ which represents that the passengers are satisfied with the quality of passenger transport service of high-speed rail.

\section{CONCLUSION}

The quality of passenger transport service of highspeed rail is a very significant influencing factor for the travel effect and how to evaluate it is a difficult problem. This study established synthetic extension evaluation model of the quality of passenger transport service of high-speed rail. The results of the example show that the model has good practicability and maneuverability.

\section{ACKNOWLEDGMENT}

This study was supported by Fundamental Research Funds of for Central Universities (grant no. 2011QNZT063).

\section{REFERENCES}

Cao, C.M. and J.J. Chen, 2012. Analysis on service quality, passenger satisfaction and passenger loyalty relationship of high-speed rail. J. China Railway Soc., 34: 1-6.

Feng, S.R., 2011. Research on the evaluation of the quality of passenger transport service of zhengxi high-speed rail and its policy. Res. Rail Econ., 2: 42-45.

Hitoshi, I., Y. Kanayama, M. Ota, T. Yamazakid and T. Okamura, 2010. How can the quality of rail services in Tokyo be further improved. Transp. Polic., 8(2): 97-106.

Liu, L.W., 2007. Comprehensive evaluation method of the improvement degree of the quality of passenger transport service of rail. Sci. Technol. Manage. Res., 6: 83-85.

Xuan, H.W., 2010. Research on the multiple-attribute evaluation method of quality of passenger transport service of the rail station. China Sci. Technol. Inform., 13: 278-279. 